\title{
PROCESSOS FÍSICOS ASSOCIADOS À GÊNESE DE UM VCAN ENTRE O OCEANO PACÍFICO E A AMÉRICA DO SUL EM ABRIL DE 2013
}

\author{
Vanessa Siqueira ${ }^{1}$, Michelle S. Reboita ${ }^{1}$, Lívia M. M. Dutra ${ }^{2}$ \\ ${ }^{1}$ Universidade Feral de Itajubá (Unifei) \\ ${ }^{2}$ Universidade de São Paulo (USP) \\ vanessa_asiqueira@hotmail.com
}

\section{RESUMO}

Esse estudo teve como objetivo avaliar, por meio das equações da tendência da vorticidade e da temperatura, os processos físicos associados à gênese de um vórtice ciclônico de altos níveis (VCAN) subtropical próximo à costa oeste da América do Sul $\left(35^{\circ}-40^{\circ} \mathrm{S}\right)$ entre os dias 30 de março a $1^{\circ}$ de abril de 2013. Entre os resultados tem-se que as advecções horizontais de vorticidade $(300 \mathrm{hPa})$ e temperatura $(500 \mathrm{hPa})$ são processos importantes para aprofundar o cavado em 300 hPa levando à gênese do VCAN.

\begin{abstract}
This study evaluates, by the equations of the vorticity and temperature local tendency, the physical processes associated with the genesis of a cyclonic vortex at upper levels (CVUL) near the west coast of South America $\left(35^{\circ}-40^{\circ} \mathrm{S}\right)$ from 30th March to 1st April 2013. Among the results is that the vorticity $(300 \mathrm{hPa})$ and temperature $(500 \mathrm{hPa})$ horizontal advections are important processes to the deepening of the trough at $300 \mathrm{hPa}$ and leading to the genesis of CVUL.
\end{abstract}

\section{INTRODUÇÃO}

Entre 30 de março a 06 de abril de 2013, um vórtice ciclônico de altos níveis (VCAN) subtropical (cutoff low no inglês) atuou sobre o centro-sul da América do Sul causando totais elevados de precipitação na Argentina e Uruguai. De acordo com Palmén e Newton (1969), os VCANs são centros de baixa pressão que se formam nos níveis médios e altos da atmosfera associados ao aprofundamento das ondas no escoamento de oeste.

Outra característica desses sistemas é a existência de um núcleo mais frio do que a periferia. Já Nieto et al. (2005) mencionam que o ciclo de vida de um VCAN contempla 4 estágios: (a) 
estágio de cavado em altos níveis da atmosfera, (b) estágio tear-off em que o cavado assume a aparência da letra ômega e no centro desse já existe uma circulação fechada, (c) estágio cutoff (VCAN) que corresponde à desagregação da circulação fechada do escoamento básico em altos níveis e (d) estágio final em que o centro de circulação fechada se une novamente ao escoamento básico.

Com relação ao VCAN ocorrido entre 30 de março a 06 de abril de 2013, Siqueira et al. (2013) realizaram uma análise sinótica detalhada do ciclo de vida desse sistema, porém neste estudo não investigaram os processos que contribuíram para sua formação. Assim, o objetivo do presente trabalho é entender, através das equações da tendência da vorticidade e da termodinâmica, os processos que favoreceram a gênese do VCAN entre 30 de março e 01 de abril de 2013.

\section{MATERIAIS E MÉTODOS}

Foi utilizada a reanálise do NCEP/NCAR (Kalnay et al., 1996) no cálculo dos termos das equações da tendência da vorticidade e da temperatura. Uma descrição detalhada dessas equações é apresentada em Dutra (2012).

A equação da tendência da vorticidade relativa (ETV) inclui os termos: variação local da vorticidade relativa (VLV), advecção horizontal de vorticidade relativa (AHV), advecção horizontal da vorticidade planetária (AHVP), advecção vertical da vorticidade relativa (AVV), divergência horizontal (DH; este termo inclui a DH multiplicada pela soma da vorticidade relativa e planetária) e termo de deformação (TD).

Também é mostrado na Fig. 1 o termo do resíduo (RESV) da ETV. A equação da tendência de temperatura (ETT) inclui os termos: variação local da temperatura (VLT), advecção horizontal de temperatura (AHT), movimento vertical (MV; soma do termo adiabático e da advecção vertical de temperatura) e termo diabático representado pelo resíduo (REST na Fig. 2). Esse estudo só mostra os termos mais importantes das equações.

\section{RESULTADOS}

Entre os dias 29/03/2013 às 12Z (Fig. não mostrada) e 31/03/2012 às 12Z (Fig. 1 - direita), a VLV em $300 \mathrm{hPa}$ é quase totalmente influenciada pela AHV (Fig. 1) com valores negativos (ciclônicos) no lado leste do cavado e positivos (anticiclônicos) no lado oeste do cavado. Das $12 Z$ do dia 29/03 (Fig. não mostrada) às $12 Z$ do dia 30/03 (Fig. 1 - esquerda), há uma Ciência e Natura Edição Especial 
intensificação da AHV anticiclônica a oeste do cavado que parece amplificar a crista na direção noroeste-sudeste contribuindo para a posterior segregação do cavado do escoamento de oeste (Fig. 1 - direita) e na formação do VCAN.
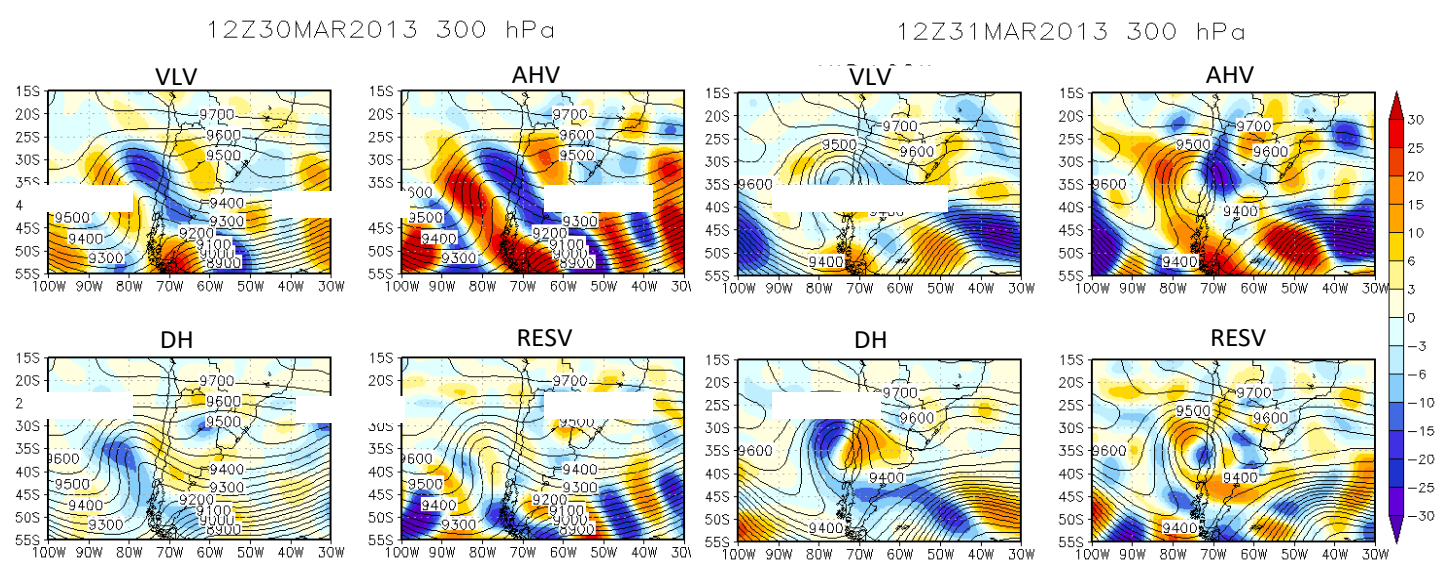

Figura 1 Altura geopotencial (linhas contínuas) e termos da ETV (sombreado): variação local (VLV), advecção horizontal (AHV), divergência (DH) e resíduo (RESV), com unidade de $10^{-10} \mathrm{~s}^{-2}$, em $300 \mathrm{hPa}$ para às $12 Z$ do dia 30 de março de 2013 (esquerda) e 12Z do dia 31 de março de 2013 (direita).

Com relação à VLT em 500 hPa, entre os dias 29/03/2013 às 12Z e 30/03/2013 às 06Z (Figs. não mostradas) a AHT domina a VLT com valores negativos (positivos) a leste (oeste) do cavado.Das $12 \mathrm{Z}$ de 30/03 às $12 \mathrm{Z}$ de 31/03 a AHT fria ocorre no centro do cavado enquanto que a quente, entre o oeste e o centro da crista localizada a oeste do cavado (Fig. 2). Temperaturas mais quentes no centro da crista e mais frias no centro do cavado contribuem para intensificar esses sistemas.

As Figuras 1 e 2 mostram que do dia 30 para o dia 31 de março, tanto em 300 quanto em 500 $\mathrm{hPa}$, os valores da altura geopotencial aumentam na crista e decrescem no centro do cavado.

Fazendo uma interpretação baseada na equação hipsométrica (não mostrada), numa coluna mais fria a pressão decresce mais rápido com a altura (o que reduz a altura geopotencial) favorecendo a intensificação da circulação ciclônica e contribuindo para a gênese do VCAN. Já numa camada quente, a pressão não decresce tão rápido. 

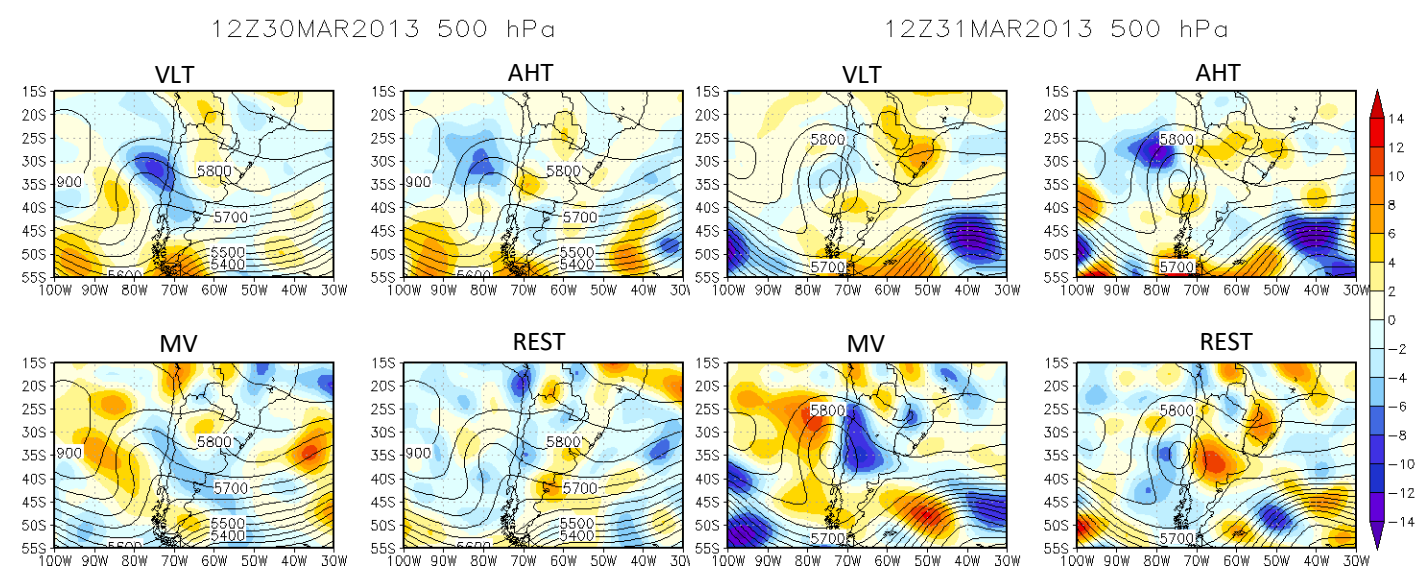

Figura 2 Altura geopotencial (linhas contínuas) e termos da ETT (sombreado): variação local de temperatura (VLT), advecção horizontal (AHT), movimento vertical (MV) e resíduo ou termo diabático

(REST), com unidade de $\mathrm{K}_{\text {dia }}^{-1}$, em 500 hPa para às $12 Z$ do dia 30 de março de 2013 (quadro da esquerda) e 12Z do dia 31 de março de 2013 (quadro da direita).

\section{CONCLUSÕES}

A análise da ETV e da ETT indica que a gênese do VCAN está associada com a AHV em $300 \mathrm{hPa}$ e AHT em $500 \mathrm{hPa}$. A atuação conjunta desses processos contribui para a amplificação da crista a oeste do cavado e, consequentemente, para a segregação desse do escoamento de oeste e gênese do VCAN.

Esse resultado concorda com Godoy et al. (2011) que observaram a AHV anticiclônica e a AHT positiva como principais processos para a formação de um VCAN na América do Sul em março de 2007.

\section{REFERÊNCIAS}

DUTRA, L. M. M. Ciclones subtropicais sobre o Atlântico Sul: análise da estrutura dinâmica de eventos. 2012. 144 f. Dissertação (Mestrado) - Instituto de Astronomia, Geofísica e Ciências Atmosféricas, Universidade de São Paulo, São Paulo.

GODOY, A. A. et al. A cut-off low in southern South America: dynamic and thermodynamic processes. Revista Brasileira de Meteorologia, v. 26, n. 4, p. 503-514, 2011.

KALNAY, E. et al. The NCEP/NCAR 40-year reanalysis project.Bulletin of the American meteorological Society, v. 77, n. 3, p. 437-471, 1996.

Ciência e Natura Edição Especial

VIII Workshop Brasileiro de Micrometeorologia 
NIETO, R. et al. Climatological features of cutoff low systems in the Northern Hemisphere. Journal of climate, v. 18, n. 16, p. 3085-3103, 2005.

PALMÉN, E.; NEWTON, C. W. Atmospheric circulation systems: their structure and physical interpretation. New York: Academic Press, 1969.

SIQUEIRA, V. A.; REBOITA, M. S.; DAUHUT, T. Chuvas na Argentina entre os dias 01 e 04 Abril de 2013 associadas a um VCAN. In V Simpósio Internacional de Climatologia, Florianópolis, 2013. 